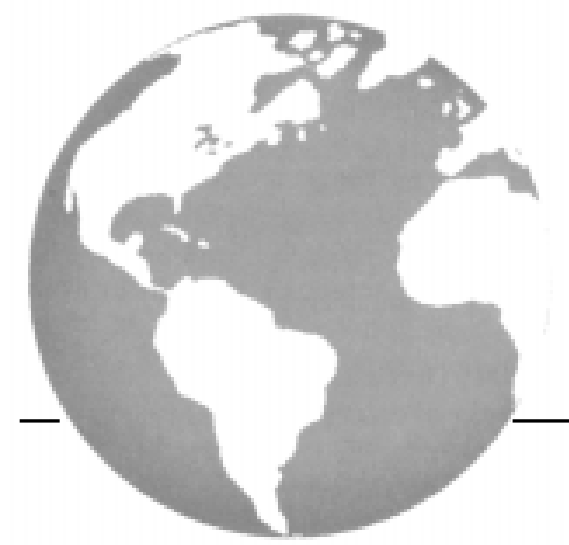

\title{
Psychological, physical, social, and economic impact of travelling great distances for cancer treatment
}

\author{
N. Lightfoot PhD, * S. Steggles PhD, ${ }^{\dagger} D$. Gauthier- \\ Frohlick MA, R. Arbour-Gagnon MA, * M. Conlon MSc, * \\ C. Innes $B A,{ }^{\S}$ L. O’Bonsawin, ${ }^{\S}$ and H. Merali*
}

\section{INTRODUCTION}

Although equity of access to cancer treatment ideally should occur regardless of residence ${ }^{1,2}$, in reality equitable access often is not the case. Despite the existence of a publicly funded health care system in Canada, not all aspects of care and travel for care are reimbursed, and claiming reimbursement is sometimes challenging ${ }^{3}$.

Cancer patients in general have additional physical, social, spiritual, and emotional requirements, and they may experience pronounced physical symptoms, social isolation, and feelings of spiritual abandonment, sadness, anxiety, deception, helplessness, anger, and guilt ${ }^{4}$. The emotional and economic impacts of a cancer diagnosis are difficult enough to handle without the added burden of having to travel long distances away from home for cancer care with or without family, friends, neighbours, or other social support networks ${ }^{5,6}$. Furthermore, residents of northeastern Ontario have the added difficulty of sometimes having to cope with very inclement weather for several winter months.

Cancer Care Ontario has aimed to deliver as much cancer care as possible close to home using local resources, and local care has been possible for many northeastern Ontario residents who require chemotherapy. However, for radiation therapy and some other forms of specialized treatment, long-distance travel to regional or central cancer treatment centres is still necessary for some patients. This travel can lead to additional emotional and economic burdens for patients and their families.

Centralization of cancer care services concentrates clinical expertise and costly specialized equipment, but research regarding the impact of additional travel and of separation from social networks is lacking. In a review of the literature of the impact of travel on the treatment experiences of cancer patients, Payne et al. ${ }^{5}$ indicated that, given contradictory literature, it is premature to conclude that travel distance and difficulty enhance psychological distress and create barriers that reduce treatment compliance and uptake.
However, they further emphasized that many cancer patients report travel for cancer treatment to be both inconvenient and adverse. Further, the patients believe that, rather than absolute distance travelled, the nature and type of travel, comfort, flexibility, safety, cost, reliance on arranged transportation, and parking facilities are important in uptake and compliance with cancer treatment. In addition, cancer patients with dependents can be concerned about disruption to family life, to care for children or elderly family members, and to work life ${ }^{2}$. Others emphasize that duration of travel, added travel expenses, and winter driving when road conditions may be hazardous (and sometimes impassable) represent burdens for cancer patients who need to travel for cancer treatment ${ }^{7}$. Financial distress associated with travel for cancer care has been documented $^{7-9}$. It is troubling that some patients decide not to undergo cancer treatment when transportation to treatment facilities is available ${ }^{10}$.

Some investigators have actually found that anxiety levels are higher for caregivers than for the cancer patients ${ }^{11}$, such that the needs of the caregivers must also be addressed by supportive care personnel and agencies that provide funds for travel for cancer treatment. For caregivers, financial difficulties and family demands can be challenging ${ }^{14}$. In Australia, Hegney et al ${ }^{12}$ undertook a qualitative study of 17 people who resided in Toowoomba and who had to travel to Brisbane for radiotherapy. Besides the burden of travel, the most common theme (82\%) that arose from the study was that patients felt they were a burden on others. Other major themes included the difficulty of living in accommodation that is not one's own, the financial burden of travelling, and the lack of closeness to family and friends.

In 1985, the Northern Health Travel Grant (NHTG) was established as a permanent program in Ontario ${ }^{13}$. Northern Ontario residents referred by a northern practitioner to the nearest physician, specialist, or health facility in Ontario or Manitoba are eligible. The grant pays one-way transportation costs by car, minus a distance of $100 \mathrm{~km}$ (or $200 \mathrm{~km}$ if the travel destination is southern Ontario). If an escort is deemed 
medically necessary and travel is by car, the patient and escort may, if desired, share the funds. Based on those conditions, the present study was designed to determine the psychological, physical, social, and economic impact of having to travel long distances for cancer treatment and care in northeastern Ontario.

\section{METHODOLOGY}

\subsection{Design}

We constructed a survey questionnaire that included questions about demographics; cancer treatment centre accessed; types of cancer treatment received; frequency and modes of travel; physical, emotional, and economic impact; and social supports. The questionnaire was available in either English or French and was designed to be readable by people with a Grade 6 education level. The questionnaires were first piloted with a small sample to verify the validity of the tool.

The research team trained the manager of the Timmins Unit of the Canadian Cancer Society, and he in turn trained and monitored all staff in the Unit who were involved in the study. The staff members telephoned potential participants and then mailed the questionnaire. If the questionnaire had not been returned after 4 weeks, the staff members made follow-up calls.

\subsection{Sample}

The study was designed for clients who interacted with the Timmins Unit of the Canadian Cancer Society, which serves the Cochrane District in northeastern Ontario. Timmins is centrally located in northeastern Ontario and is approximately $680 \mathrm{~km}$ northwest of Toronto, or about $290 \mathrm{~km}$ northwest of Sudbury. Most clients in the Cochrane District surrounding Timmins access the Regional Cancer Program of the Hôpital régional de Sudbury Regional Hospital (HRSRH) in Sudbury, Ontario. Large employers in the District include the mining and smelting sectors; forestry, pulp, paper, and wood products; manufacturing; metal fabrication; and the service and tourism industries.

To be invited to participate in the study, cancer patients had to meet all of these criteria:

- Resident in the Cochrane District

- Age 18 years or older

- Contacted the Canadian Cancer Society, Timmins Unit, during July - September 1999 or September - October 2000

- Had to travel out of the District for cancer treatment one or more times

The 410 patients who met these criteria were telephoned by the Timmins Unit staff, who used standard invitation wording to invite participation in the study. Eligible patients were those who self-com- pleted the questionnaire and who were age 18 years or older at the time of questionnaire completion. Unit staff were trained in advance and were monitored by the Unit manager, who consulted extensively with the study co-investigators. If a completed questionnaire was not received within 4 weeks, an additional questionnaire was mailed.

\subsection{Analysis}

Quantitative data was entered into and analyzed using the SPSS software program, version 11.5 (SPSS, Chicago, IL, U.S.A.).

\subsection{Ethics}

The study underwent ethical review by the Research Ethics Committee at the HRSRH. During the study period, a total of 410 questionnaires were mailed to eligible study subjects who agreed to participate in the study. The 133 returned questionnaires represent a response rate of $32.4 \%$.

\section{RESULTS}

\subsection{Demographics}

Most respondents were female (65.4\%), of FrenchCanadian ethnicity (49.6\%) [closely followed by English-Canadian ethnicity (42.9\%)], 65 years of age or younger $(57.1 \%$; mean: $60.4 \pm 13.8$ years; range: $18-88$ years), resident in Timmins and South Porcupine $(81.2 \%)$, and in married or common-law relationships (61.7\%). Among all respondents, 39.8\% had a secondary school education, $33.8 \%$ had elementary or no education, and $24.1 \%$ had a college or university education; $33.8 \%$ were retired, and $12.8 \%$ worked full-time; $75.2 \%$ had two or more children, and $80.2 \%$ had children 19 years of age or older.

\subsection{Choices of Treatments}

Most participants accessed their cancer treatments at the Regional Cancer Program of the HRSRH in Sudbury (70.2\%), followed by Princess Margaret Hospital in Toronto (10.6\%), and the Northwestern Ontario Regional Cancer Centre in Thunder Bay $(5.0 \%)$. Of these respondents, $25.5 \%$ received radiation therapy, $15.8 \%$ chemotherapy, and $21.4 \%$ surgery. The remainder received other therapies or had follow-up appointments. The top five cancer sites for these respondents were reported to be (from highest to lowest) breast $(30.8 \%)$, lung $(8.3 \%)$, prostate $(6.8 \%)$, throat $(5.3 \%)$, and colon $(3.8 \%)$.

Approximately half of the respondents were able to choose their cancer treatment centre. For most respondents, their physician had recommended a particular treatment centre $(78.2 \%)$. Only $14.3 \%$ indicated that the travel prospects influenced their 
choice of treatment, although $27.8 \%$ indicated that they did not have treatment choices, and $26.3 \%$ noted that they had no choice but to travel for treatment.

\subsection{Physical Impact}

More than half of the respondents reported experiencing physical symptoms as a result of travelling to a cancer centre, and $28.6 \%$ reported discomfort ranging from "a fair amount" to "very much." The patients were usually accompanied to the cancer centre by an escort, the most usual escort being a spouse $(60.9 \%)$; $17.5 \%$ reported driving themselves for visits or treatments. The mean one-way distance travelled by patients to a cancer centre was $396.24 \pm 169.79 \mathrm{~km}$ (range: $160-850 \mathrm{~km}$ ). The most frequent mode of travel was travel by car $(75.6 \%)$. Most respondents $(61.7 \%)$ had to travel for cancer treatment during the winter, and most of these found winter travel to be difficult or very difficult $(68.3 \%)$. Most stayed in either a cancer centre lodge or a hotel while accessing cancer treatment $(88.2 \%)$. Only $17.4 \%$ of respondents reported having difficulty parking at the cancer centre.

\subsection{Economic Impact}

The average round-trip cost for travel by car for cancer treatment was not excessive, but costs varied widely depending on the distance travelled. Of the respondents, $93.2 \%$ normally applied for financial assistance provided by the NHTG, and most had never had a travel grant rejected $(79.7 \%)$. However, about half felt that the level of financial assistance required improvement or major improvement $(54.1 \%)$; and just a quarter $(25.6 \%)$ indicated that they experienced no difficulties in meeting the financial demands of their travel.

More than half of respondents $(61.7 \%)$ reported that travelling for cancer treatments affected their ability to carry out their job or schoolwork, and almost a quarter $(23.4 \%)$ reported that travelling totally prevented them from doing their job. Household duties are also greatly compromised for a great number of patients, with $15.0 \%$ reporting severe difficulties in completing chores.

The average cost of a round trip by car varied widely, with the mean being $\$ 167.61 \pm \$ 141.54$. Only a small number of study participants had never applied for an NHTG, and only $13.5 \%$ had ever been rejected. More than half of the respondents $(54.1 \%)$ listed the overall financial assistance as "not satisfactory," "requires improvement," or "requires major improvement," and $42.9 \%$ reported "a fair amount," "much," or "very much" difficulty in meeting the financial demands of travel for treatment.

\subsection{Psychological and Social Impacts}

Most respondents reported emotional difficulty associated with travel from home to a cancer centre
$(80.5 \%) ; 18.0 \%$ reported having no emotional difficulty (Table I). Similarly, $76.8 \%$ of spouses and $78.5 \%$ of families also found travel from home to a cancer centre to be emotionally difficult.

We used logistic regression modelling to identify factors associated with greater emotional difficulty for travel from home to a cancer centre. People who were married or in common-law relationships $(p=0.033)$ and who were 65 years of age or younger $(p=0.011)$ reported significantly greater emotional difficulty. The sex of these patients, availability of an escort, winter travel, distance travelled, and education were not associated with emotional difficulty (Table II). People over the age of 65 reported significantly less emotional difficulty associated with travel for cancer treatment than that reported by patients 65 years of age or younger. The sex of these patients, availability of a travel escort, winter travel, distance travelled, and education were not associated with emotional difficulty.

\subsection{Qualitative Data}

\subsubsection{Methodology}

We analyzed the qualitative data using the NUD*IST 5 software program (QSR International, Doncaster, Victoria, Australia).

In addition to the questions that asked respondents about the level of physical difficulty associated with travel to a cancer centre, responses to open questions provided data for qualitative analyses. We used the "immersion and crystallization" qualitative method to extrapolate emerging themes. This method consists of the analyst's prolonged immersion into and experience of the text and then an emergence, after concerned reflection, with an "intuitive crystallization of the data." We used this technique to identify themes reflecting the predominant perspectives evident in the responses ${ }^{14}$. Predominant themes included comments about fatigue, weakness, pain, discomfort, emotional distress, travel and road conditions, conditions within the vehicle, and requirements for travel.

\subsection{Qualitative Analysis}

\subsubsection{Funding}

Themes concerning financial issues predominated. Respondents rated the level of financial assistance that they received for travelling to a cancer centre and suggested increases in the NHTG to cover all travel expenses. Respondents reported that more information is needed about the NHTG-for example, how to appeal when denied funding.

The Canadian Cancer Society assists patients and their families with transportation and often with funding. Volunteer drivers are very much appreciated, as indicated by comments such as this: "Travelling with the CCS Volunteer Drivers has been a positive expe- 
TABLE I Personal impact of travel for cancer treatment

Statement

Not at all A little A fair amount Much Very much Missing

Travel from home to cancer centre emotionally difficult $(n=133)$

$\begin{array}{cc}18.0 & 27.8 \\ (n=24) & (n=37)\end{array}$

Spouse found travel from home to cancer centre emotionally difficult $(n=99) \quad \begin{array}{ll}17.2 & 20.2\end{array}$

$(n=17) \quad(n=20)$

15.0

15.0
$(n=20)$

19.2
$(n=19)$

$13.5 \quad 24.1 \quad 1.5$

$\begin{array}{ccc}13.5 & 24.1 & 1.5 \\ (n=18) & (n=32) & (n=2)\end{array}$

$(n=19) \quad(n=12) \quad(n=25) \quad(n=6)$

Family found your travel from home to cancer centre emotionally difficult $(n=130)$

Travel to cancer centre affected relationship with spouse $(n=102)$

Travel to cancer centre affected relationship with family $(n=133)$

Travel to cancer centre affected relationship with friends $(n=132)$

$\begin{array}{cccccc}16.2 & 26.2 & 17.7 & 12.3 & 22.3 & 5.4 \\ (n=21) & (n=34) & (n=23) & (n=16) & (n=29) & (n=7) \\ 52.9 & 19.6 & 8.8 & 2.0 & 6.9 & 9.8 \\ (n=54) & (n=20) & (n=9) & (n=2) & (n=7) & (n=10) \\ 61.7 & 15.0 & 9.0 & 4.5 & 3.8 & 6.0 \\ (n=82) & (n=20) & (n=12) & (n=6) & (n=5) & (n=8) \\ 71.2 & 13.6 & 6.1 & 1.5 & 2.3 & 5.3 \\ (n=94) & (n=18) & (n=8) & (n=2) & (n=3) & (n=7) \\ & & & & & \end{array}$

TABLE II Multivariate odds ratios estimates and 95\% confidence intervals (CIs) for factors associated with emotional difficulty from travel $(n=120)$

\begin{tabular}{llclc}
\hline Factor $^{a}$ & Group & More emotion $^{b}$ & Less emotion $^{c}$ & Odds ratio $\left(95 \%\right.$ CI $^{\circ}$ \\
\hline Marital status & Single, widowed, divorced, separated & 18 & 28 & \\
& Married, common-law & 46 & 28 & $2.63(1.08-6.37)$ \\
Age group & 65 years and under & 47 & 28 & 28 \\
& Over 65 years & 17 & $0.31(0.12-0.76)$ \\
\hline
\end{tabular}

a Not retained in model: escort (yes/no), sex (male/female), winter travel (yes/no), distance $(\leq 400 \mathrm{~km}$ versus $>400 \mathrm{~km})$, and education (elementary or no formal education versus others).

b For example, respondents found travel from home to cancer centre "fairly," "much," or "very much" emotionally difficult.

c For example, respondents found travel from home to cancer centre "not at all" or "a little" emotionally difficult.

rience for me. The drivers were courteous, helpful, and friendly and all should be commended for their great generosity in providing such a needed service." Another example was this: "Without the Cancer Society's volunteer service, travelling would have been much more expensive and inconvenient as time spent at home on weekends during radiation treatment would have been much reduced, [and] sometimes not feasible in winter driving conditions." Others reported that if, the NHTG could pay for each patient travelling in the van, the Canadian Cancer Society would be able to provide "a more comfortable van and only non-smoking drivers would be needed, [because] some drivers smelled strongly of smoke." Another patient commented, "Cramped condition, too many in car on some trips."

Inadequacies in the NHTG were identified repeatedly in the responses. For example, "I received only $\$ 444$ a month on 'workfare.' When I travel to the centre, most times I must borrow money to be able to eat. We should be given sufficient money for travelling cost and for meals." Others needed the assistance before they travelled: "I don't always have the funds available to travel therefore I have to borrow money from relatives. [The] travel grant should be higher than $\$ 97$ and we should be able to get the funds before going as it was in the past."
Winter travel can be costly as reflected by the comment "When travelling with your car, you have wear and tear that is very costly. I blew a gasket in the motor and the car was stuck in the garage for six weeks. Snow and rain caused me to go off the road in the ditch near the Provincial Park and I had to be towed." One patient who travelled $800 \mathrm{~km}$ to Toronto reported that "The $\$ 200$ I received for the travel grant does not cover my expenses for two days [of] travel, lodging, gas, etc. I live $800 \mathrm{~km}$ from Toronto ... It would help to give people who live over $500 \mathrm{~km}$ away a little bit more money."

The NHTG refunds were not always timely: "Cheques are late in coming. Still waiting in midJuly for [travel] in early May." Another person reported that "I have been travelling to the cancer centre for five months and have applied for a NHTG several times. One form was sent back due to missing information. Another was returned because it was missing the doctor's referral and upon calling the 1-800 number I was told that I only needed one doctor's referral in six months. I have not received any refund as of yet."

Inequality and disparity in funding reimbursement between Ontario's north and south was also mentioned. For example, "Northerners are not allowed the same travel treatment as the South. This is 
why I make my own way for flights or car travel and hotel arrangements. I am not refunded in full. I always have to appeal my case, and then only get a partial travel grant, as for others in the South they get the full travel grant and allowances not given to us in the North."

\subsubsection{Emotional Impact}

Winter travel and road conditions were reported to be very stressful for patients and their families. Illustrative comments included these: "I need company, I am afraid of travelling alone for eight hours." Some people experienced "discomfort while driving," and they found roads "slippery and isolated during winter" travel. Many who travelled great distances for treatment reported emotional distress: "I was tired, very weak, and I found it very stressful." Others also experienced fatigue and weakness: "I was tired, stressed by weather, and others in the vehicle." Also, "Everything went very well. The only thing, it was very tiresome and travelling, especially after having treatments you are weak. Being a senior could also be the cause."

Travelling may also cause discomfort or pain: "After treatments, I find it hard to travel because of side effects. Had choking attacks along (highway) 144 , had to stop instantly and try to catch [my] breath, and couldn't drive."

Others commented on volunteers. For example, "All the volunteers were like angels for us and the staff were always there for us, no matter what we needed," and "great care and consideration [were provided] by volunteer drivers and staff at the lodge and cancer centre."

\subsection{Study Limitations}

The response rate for this study was much lower than desired. As a result, the study may not be generalized for cancer patients and for those who must travel long distances for cancer treatment. In a future study, it would be interesting to evaluate and compare the impacts on the escort or partner who travels with the cancer patient.

\section{DISCUSSION}

Respondents to our questionnaire reported physical and emotional symptoms associated with long distance travel for cancer treatments. When factors associated with greater emotional difficulty were examined, an age of 65 years or younger and an existing married or common-law relationship were significantly associated with greater emotional difficulty. Younger patients may be more worried about the effect of their diagnosis, treatment, travel, possibly premature outcome, and employment disruption for their families and employers. Similarly, cancer patients who have significant others, and possibly families, may be quite concerned about the same effects and issues.

There is no way of knowing whether the results of this study can be extended beyond the direct participants. However, it is quite possible that they can and that Ontario cancer patients and their caregivers who must travel long distances for cancer treatment could benefit from routinely being offered supportive care counselling to reduce the emotional difficulty experienced while travelling for care. If additional financial resources need to be allocated for additional supportive care personnel to assist cancer patients who must travel long distances for treatment at cancer centres, it would be money well spent. Additional telephone counselling could also be given to families and patients, if desired.

With the advent of the new Northern Ontario School of Medicine, students will spend placements in some of the smaller communities where cancer patients who travel for treatment reside. The students may be able to assist in supportive care counselling for some patients. Furthermore, with the advent of the presence of nurse practitioners in some of the smaller and rural and remote communities, supportive care counselling for cancer patients could also be part of the nursing mandate. It will be important to evaluate the effectiveness of clinical and supportive care, and the satisfaction provided to patient, family, and escort by these various health care professionals in various settings (for example, social workers in a cancer centre, psychologists in a cancer centre, family physicians within the home community, psychologists within the home community, psychiatrists within the home community, medical school practicum students within the home community, nurse practitioners within the home community, and so on).

Several issues were raised about NHTG funding and about the services available through the Canadian Cancer Society. Not all respondents were aware of the process involved in the application of these services. Respondents welcomed volunteer drivers to transport them for their treatments, emphasizing the importance and role of volunteer drivers from the Canadian Cancer Society.

More than half of the respondents were accompanied to a cancer centre by an escort who often was a spouse or secondarily a child or a parent. Travelling for treatments is more difficult in the winter or when the patient is alone.

In November 2001, revisions were made to the NHTG to provide funding for two-way (as opposed to one-way) travel to the nearest cancer specialist, minus $100 \mathrm{~km}$ (or minus $200 \mathrm{~km}$ if the travel is to southern Ontario); no additional reimbursement is made for meals and accommodation ${ }^{15}$. This change is something of an improvement, but the arrangements could still leave some cancer patients with large financial expenses and emotional isolation. During the study, some participants made comments indicating that the 
NHTG criteria were not always consistently applied. Although we have no way to verify the relevant facts, it would be helpful to ensure that NHTG criteria are consistently applied.

During the study, several cancer patients from northern Ontario mentioned that breast and prostate cancer patients from southern Ontario who were temporarily referred to northern Ontario because of unacceptable treatment wait times received full funding for various modes of travel, plus meals and accommodation. This more comprehensive funding was provided through a temporary program, the Radiation Re-referral Program, that began in the spring of 1999. That program was available to all breast and prostate cancer patients in Ontario who had to be referred to centres other than their home centre because of unacceptable radiation wait times (beyond 8 weeks). Unacceptable wait times were not then experienced at northern Ontario cancer centres. The Radiation Re-referral Program paid for two-way car travel, meals, and accommodation (within limits) for patients. It did not reimburse escort costs. In May 2001, the Ontario Ombudsman ruled the funding discrepancy to be "improperly discriminatory," and the program ceased to exist in the spring of 2001.

Under the present NHTG criteria, northern Ontario cancer patients who have lower incomes and who must travel long distances for treatment could still face financial difficulties in addition to coping with a potentially life-threatening disease. An even more serious situation may develop when an employed cancer patient either has to take medical leave from work or to permanently leave employment. Furthermore, routine payment for medical escort costs could be helpful for all cancer patients who must travel long distances for treatment. Having the support of a family member or friend could be very important to the emotional health of the cancer patient.

More and more, chemotherapy is being performed in local hospital facilities, rather than only in cancer centres. However, radiotherapy is delivered only in cancer centres and will continue to be so delivered because of the cost of the specialized equipment to a publicly funded health care system. For these reasons, additional financial and policy changes remain important to Ontario cancer patients.

Cancer Care Ontario drafted a strategy to reduce waiting times for cancer services in Ontario ${ }^{16}$. As part of that strategy, the Cancer Quality Council of Ontario proposed the following four-point approach to dealing with waiting times for cancer services:

1. Reduce demand for services by reducing risk of cancer development and by promoting early detection.

2. Enhance the supply of cancer resources.

3. Coordinate access to cancer services.

4. Increase efficient use of current cancer resources.
The methods for achieving the foregoing goals that are applicable to the findings of the present study are these:

- Target expansion of cancer system resources according to need.

- Expand the roles and skill mix of health care professionals to enhance the capacity of the system.

- Coordinate the patient journey from diagnosis onwards.

- Establish diagnostic assessment units and rapid access models for improved access to appropriate diagnostic services.

- Improve processes and cycle times.

- Ensure that cancer patients are treated in a manner consistent with evidence for best practice.

In Ontario, Fitch et al. ${ }^{17}$ found these themes arising from interviews with those who travel for cancer radiation treatment:

- Waiting time represents the most difficult part of the process.

- The idea of travelling for treatment is distressing.

- Travelling for treatment leads to fatigue and creates difficulties for patients.

- Being away from home has advantages and disadvantages.

Fitch and colleagues also recommended that supportive care strategies be developed and implemented for cancer patients who need to travel. This recommendation will lead to greater allocation of funds to supportive cancer care programs in Ontario.

The present study emphasizes that travel is an even greater difficulty for those who reside in northern Ontario, where travel for cancer treatment is a frequent necessity. It will be important to evaluate proposed supportive care interventions for patients, their families, and their significant others not only from a social, political, and ethical perspective, but also (in light of a more cost conscious health care environment) from an accountability and economic perspective ${ }^{4}$. However, funding, availability of supportive care personnel to assist cancer patients and their families who must travel for cancer treatment, and coordinated access to cancer services can still be enhanced further in Ontario.

\section{CONCLUSION}

Many issues of importance similar to those described in this study arise for cancer patients who travel long distances for cancer care within and between developed countries. Cancer patients from northern Ontario may benefit from additional financial and emotional support. Financial support programs may be better received when applied consistently for various geographic areas. The availability of supportive 
care personnel to assist and support cancer patients and their families who must travel for cancer treatment, and coordinated access to cancer services, can still be enhanced further in Ontario.

The results of this study are invaluable for planners from hospitals, cancer centre programs, and the Canadian Cancer Society. They can be used to improve the travel-related experiences of those who must travel long distances for cancer treatment.

Many of the results of this study are consistent with those observed in a Canadian Cancer Society study of cancer patient and caregiver needs in Ontario ${ }^{15}$.

\section{ACKNOWLEDGMENTS}

We are most grateful for financial support for this study from the Canadian Cancer Society and the Northern Cancer Research Foundation.

\section{REFERENCES}

1. The Expert Advisory Group on Cancer to the Chief Medical Officers of England and Wales. A Policy Framework for Commissioning Cancer Services [Calman Hine Report]. London: Department of Health; 1995.

2. Payne S, Jarrett N, Jeffs D, Brown L. Implications of social isolation during cancer treatment. The implications of residence away from home during cancer treatment on patients' experiences: a comparative study. Health Place 2001;7: 273-82.

3. Davis C, Girgis A, Williams P, Beeney L. Needs assessment of rural and remote women travelling to the city for breast cancer treatment. Aust N Z J Public Health 1998;22:525-7.

4. Szucs TD. Medical economic considerations of supportive cancer care. Int J Antimicrob Agents 2000;16:181-4.

5. Payne S, Jarrett N, Jeffs D. The impact of travel on cancer patients' experiences of treatment: a literature review. Eur $J$ Cancer Care (Engl) 2000;9:197-203.

6. Baider L, Kaufman B, Ever-Hadani P, Kaplan De-Nour A. Coping with additional stresses: comparative study of healthy and cancer patient new immigrants. Soc Sci Med 1996;42: 1077-84.

7. Meden T, St. John-Larkin C, Hermes D, Sommerschield S. Relationship between travel distance and utilization of breast cancer treatment in rural northern Michigan. JAMA 2002; 287:111.

8. McGrath P. Returning home after specialist treatment for hematological malignancies: an Australian study. Fam Cоттиnity Health 2001;24:36-48.
9. Crooks DL, Whelan TJ, Reyno L, et al. The Initial Health Assessment: an intervention to identify the supportive care needs of cancer patients. Support Care Cancer 2004;12:19-24.

10. Guidry JJ, Aday LA, Zhang D, Winn RJ. Transportation as a barrier to cancer treatment. Cancer Pract 1997;5:361-6.

11. Clavarino AM, Lowe JB, Carmont SA, Balanda K. The needs of cancer patients and their families from rural and remote areas of Queensland. Aust J Rural Health 2002;10:188-95.

12. Hegney D, Pearce S, Rogers-Clark C, Martin-McDonald K, Buikstra E. Close, but still too far. The experience of Australian people with cancer commuting from a regional to a capital city for radiotherapy treatment. Eur J Cancer Care (Engl) 2005;14:75-82.

13. Ontario, Ministry of Health and Long-Term Care. Public Information, Publications, OHIP, Northern Health Travel Grant Program [Web page]. Toronto: Queen's Printer for Ontario; 2002. [Available at: http://www.health.gov.on.ca/english/public/pub/ohip/northern.html; cited June 30, 2005]

14. Crabtree BF, Miller WL. Doing Qualitative Research. 2nd ed. Thousand Oaks, CA: Sage Publications; 1999.

15. Canadian Cancer Society. Breaking Down the Barriers: Study of Cancer Patient and Caregiver Needs in Ontario. Toronto: Canadian Cancer Society; 2003.

16. Schwartz F, Evans W, Angus H. A Four-Point Strategy to Reduce Waiting Times in Ontario. Toronto: Cancer Quality Council of Ontario; 2004.

17. Fitch MI, Gray RE, McGowan T, et al. Travelling for radiation cancer treatment: patient perspectives. Psychooncology 2003;12:664-74.

Corresponding author: Nancy Lightfoot, Hôpital régional de Sudbury Regional Hospital, 41 Ramsey Lake Road, Sudbury, Ontario P3E 5J1.

E-mail: nlightfoot@hrsrh.on.ca

* Epidemiology Research Unit, Regional Cancer Program of the Hôpital régional de Sudbury Regional Hospital, Sudbury, Ontario.

$\dagger \quad$ Psycho-Oncology Services: Education, Clinical, and Research, St. Luke's Hospital, Dublin, Ireland.

¥ Supportive Care Oncology Research Unit, Regional Cancer Program of the Hôpital régional de Sudbury Regional Hospital, Sudbury, Ontario.

$\S$ Canadian Cancer Society, Sudbury, Ontario. 